\title{
Prognostic Significance of Homocysteine Level on Neurological Outcome in Brain Arteriovenous Malformations
}

\author{
Fa Lin $\mathbb{D}^{1,2,3,4}$ Chaofan Zeng $\mathbb{D}^{1,2,3,4}$ Peicong Ge $\mathbb{D}^{1,2,3,4}$ Dong Zhang, ${ }^{1,2,3,4}$ \\ Shuo Wang $\mathbb{D}^{1,2,3,4}$ and Jizong Zhao $\mathbb{i}^{1,2,3,4,5}$ \\ ${ }^{1}$ Department of Neurosurgery, Beijing Tiantan Hospital, Capital Medical University, No. 119 South Fourth Ring Rd West, \\ Fengtai District, Beijing 100070, China \\ ${ }^{2}$ China National Clinical Research Center for Neurological Diseases, Beijing 100070, China \\ ${ }^{3}$ Center of Stroke, Beijing Institute for Brain Disorders, Beijing 100070, China \\ ${ }^{4}$ Beijing Key Laboratory of Translational Medicine for Cerebrovascular Disease, Beijing 100070, China \\ ${ }^{5}$ Savaid Medical School, University of the Chinese Academy of Sciences, Beijing, China \\ Correspondence should be addressed to Jizong Zhao; zhaojz205@163.com
}

Received 15 October 2020; Revised 9 November 2020; Accepted 11 November 2020; Published 30 November 2020

Academic Editor: Wen-Jun Tu

Copyright (C) $2020 \mathrm{Fa}$ Lin et al. This is an open access article distributed under the Creative Commons Attribution License, which permits unrestricted use, distribution, and reproduction in any medium, provided the original work is properly cited.

\begin{abstract}
Objective. We aimed to investigate the serum homocysteine (Hcy) level in patients with brain arteriovenous malformation (bAVM) and their impact on neurological outcome during hospitalization. Method. We retrospectively reviewed patients diagnosed with bAVMs in Beijing Tiantan Hospital from January 2019 to August 2020. Patients were divided into two groups according to the mRS (modified Rankin Scale) score at discharge. Clinical and laboratory characteristics were compared. Logistic regression analyses were performed to identify the potential risk factors for short-term neurological outcome. Results. A total of 175 bAVM patients were enrolled in the study, including 139 patients with favorable outcome $(\mathrm{mRS} \leq 2)$ and 36 patients with unfavorable outcome $(\mathrm{mRS}>2)$. Hyperhomocysteinemia was identified in $32.6 \%$ of cases $(n=57)$. Serum Hcy level was related to seizure manifestation $(P=0.034)$ and short-term neurological outcome $(P=0.027)$. Logistic regression analysis showed that serum glucose (OR 1.897, 95\% CI 1.115-3.229; $P=0.018)$ and Hcy level (OR 0.838, 95\% CI 0.720-0.976; $P=0.023$ ) were significantly associated with short-term disability. Conclusion. Our results indicated that the lower serum Hcy level is strongly associated with in-hospital unfavorable outcome. Further prospective studies of Hcy natural history and managements in bAVMs are required, which would be valuable for evaluating the disease-modifying efficacy of oral nutritional supplements in bAVM patients.
\end{abstract}

\section{Introduction}

Brain arteriovenous malformations (bAVMs) are well known as congenitally abnormal conglomerations of dilated feeding arteries and draining veins without intervening capillary beds $[1,2]$. Although bAVM occurs in approximately $15 / 100,000$ persons, it is still the leading cause of hemorrhagic stroke in young people and often results in a high incidence of neurological morbidity and mortality [2]. Prediction of outcomes is mostly based on demographic, clinical, and radiographic markers, while the information on routine laboratory examinations is inadvertently ignored.

Hyperhomocysteinemia (HHcy) is an elevation of serum homocysteine (Hcy) concentration due to the methionine metabolic disorder [3]. Characterized by the atherogenic and thrombogenic effects, Hcy is recognized as the independent risk factor for the major adverse cardiovascular and cerebrovascular events (MACCEs) and mortality $[4,5]$. Apparently, previous studies have focused on hemorrhagic stroke and unfavorable outcomes $[5,6]$. Moreover, some studies suggested that Hcy disorders and hemorrhagic bAVMs shared similar underlying mechanisms in terms of hemodynamics [7-9] and molecular abnormalities [10-14]. However, the above-mentioned studies were based on other hemorrhagic subtypes and animal experiments; the clinical significance of Hcy in patients with bAVM has not been discussed yet.

The present study was investigated to take an exploratory look into the association between serum Hcy levels in 
patients with bAVM and their impact on neurological outcome during hospitalization.

\section{Materials and Methods}

2.1. Study Design and Participants. We retrospectively reviewed patients diagnosed with bAVMs at the Department of Neurosurgery, Beijing Tiantan Hospital, from January 2019 to August 2020. The study was approved by the Institutional Review Board of our institution, and informed consent was waived considering the retrospective design of the study.

Among 402 patients with cerebral vascular malformations admitted to our hospital between January 2019 and August 2020, 332 patients were diagnosed with bAVMs using digital subtraction angiography (DSA) or magnetic resonance imaging (MRI). Patients with inadequate laboratory or DSA data were excluded. Finally, a total of 175 patients were included in the study (Figure 1). The enrolled patients were divided into two groups according to the neurological outcome at discharge (Group 1, modified Rankin Scale [mRS] $\leq 2$; Group 2, mRS $>2$ ).

2.2. Data Collection and Outcome Evaluation. Demographic data, medical and personal history, prior treatments, primary symptoms, bAVM radiographic characteristics, clinical features, laboratory results, and treatment modality were obtained. Medical and personal history including hypertension, diabetes mellitus, hyperlipidemia, cigarette smoking, and alcohol drinking were recorded. The treatment history included embolization, radiosurgery, and microsurgery. Primary symptoms were summarized as hemorrhage, seizure, and neurological dysfunction. Radiographic characteristics included volume of lesion, deep and eloquent location, venous drainage patterns, and associated aneurysms. The AVM volume was calculated by $(a \times b \times c) / 2$. SpetzlerMartin (SM) grading scale was evaluated to stratify the bAVMs. The treatment modalities were dichotomized based on the involvement of microsurgery. The clinical status was determined by the mRS score at admission and discharge. Neurological disability $(\mathrm{mRS}>2)$ was defined as the clinical outcome. The neurological assessment at discharge was considered a short-term outcome.

Heart rate and blood pressure were also obtained. Body mass index (BMI) was calculated as weight $(\mathrm{kg}) /[\text { height }(\mathrm{m})]^{2}$. Fast venous blood samples were collected in the morning after admission for all bAVM patients. Levels of blood glucose, albumin (ALB), creatinine, uric acid (UA), total cholesterol (TC), triglyceride (TG), high-density lipoprotein cholesterol (HDL-C), low-density lipoprotein cholesterol (LDL-C), apolipoprotein A (ApoA), apolipoprotein B (ApoB), and Hcy were measured using enzymatic methods. Serum $\mathrm{Hcy} \geq 15.0$ $\mu \mathrm{mol} / \mathrm{L}$ was defined as HHcy [15].

2.3. Statistical Analysis. Categorical variables were expressed as frequencies, and continuous variables were presented with a mean (standard deviation (SD)) or median (interquartile range (IQR)). A chi-square test or Fisher's exact test was performed to compare categorical variables between groups. Continuous variables were compared by two-tailed Student's

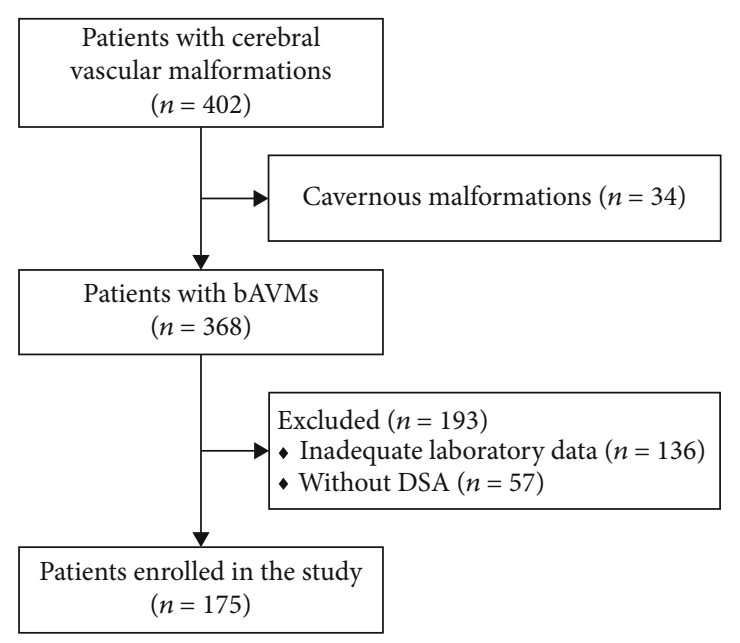

FIGURE 1: Flow diagram of the study participants. bAVMs: brain arteriovenous malformations; DSA: digital subtraction angiography.

$t$-test or Mann-Whitney $U$ test. The association between variables and Hcy quartiles was analyzed using the Cochran-Armitage test for bivariate variables and Spearman's rank correlation test for continuous variables. Logistic regression analyses were conducted to identify the potential risk factors for short-term neurological outcome. Variables achieving $P<0.10$ in univariate analysis were included in the multivariate analysis. $P$ value $<0.05$ was considered statistical significance. Statistical analyses were performed using SPSS 26.0 (IBM, New York, USA).

\section{Results}

Four hundred and two cerebral vascular malformation patients were identified. After excluding 34 cavernous malformations and 193 patients with incomplete data, a total of $175 \mathrm{bAVM}$ patients were enrolled in our study (Figure 1).

3.1. Baseline Characteristics of bAVM Patients. The baseline characteristics of bAVM patients are shown in Table 1. The mean age at diagnosis was 29.6 years, with a male-to-female ratio of $1.3: 1$. Thirty-one cases $(17.7 \%)$ were at cigarette smoking status, and 17 cases (9.7\%) were current alcohol abused. Sixty cases $(34.3 \%)$ had received prior treatments, including embolization in $43(24.6 \%)$, radiosurgery in 12 (6.9\%), and microsurgery in 5 (2.9\%). Hemorrhage (56.6\%) occurred as the most common primary symptom, followed by seizure (20.0\%) and neurological dysfunction (17.7\%). Poor neurological status (mRS > 2) was observed in $15.4 \%$ of cases $(n=27)$ on admission. Twenty-eight cases $(16.0 \%)$ harbored SM grade IV-V lesions. The average volume of lesions was $8.6 \mathrm{~cm}^{3}$. Deep locations were found in 51 cases (29.1\%), and eloquent areas were involved in $52.0 \%$ of cases $(n=91) .34 .3 \%$ of cases $(n=60)$ had deep venous drainage, and $12.6 \%$ of cases $(n=22)$ had associated aneurysms. HHcy was identified in $32.6 \%$ of cases $(n=57)$. According to the treatment modalities, microsurgery was involved in 78 cases (44.6\%). Compared with Group 1, patients in Group 2 more likely presented with hemorrhage $(P=0.001)$, and exhibited 
TABLE 1: Baseline characteristics of bAVM patients between groups.

\begin{tabular}{|c|c|c|c|c|}
\hline Variables & Total $(n=175)$ & Group $1^{\#}(n=139)$ & Group $2^{\#}(n=36)$ & $P$ value \\
\hline Age $(y)$, mean $(S D)$ & $29.6(14.7)$ & $29.9(14.6)$ & $28.6(15.1)$ & 0.635 \\
\hline Sex, male (\%) & $100(57.1)$ & $78(56.1)$ & $22(61.1)$ & 0.589 \\
\hline \multicolumn{5}{|l|}{ Medical and personal history (\%) } \\
\hline Hypertension & $14(8.0)$ & $9(6.5)$ & $5(13.9)$ & 0.264 \\
\hline Diabetes mellitus & $5(2.9)$ & $3(2.2)$ & $2(5.6)$ & 0.597 \\
\hline Hyperlipidemia & $1(0.6)$ & $1(0.7)$ & $0(0)$ & $>0.999$ \\
\hline Cigarette smoking & $31(17.7)$ & $25(18.0)$ & $6(16.7)$ & 0.853 \\
\hline Alcohol drinking & $17(9.7)$ & $13(9.4)$ & $4(11.1)$ & 0.999 \\
\hline \multicolumn{5}{|l|}{ Prior treatments $(\%)$} \\
\hline Embolization & $43(24.6)$ & $32(23.0)$ & $11(30.6)$ & 0.349 \\
\hline Radiosurgery & $12(6.9)$ & $8(5.8)$ & $4(11.1)$ & 0.445 \\
\hline Microsurgery & $5(2.9)$ & $5(3.6)$ & $0(0)$ & 0.585 \\
\hline \multicolumn{5}{|l|}{ Primary symptom (\%) } \\
\hline Hemorrhage & $99(56.6)$ & $70(50.4)$ & $29(80.6)$ & $0.001^{*}$ \\
\hline Seizure & $35(20.0)$ & $30(21.6)$ & $5(13.9)$ & 0.304 \\
\hline Neurological dysfunction & $31(17.7)$ & $24(17.3)$ & $7(19.4)$ & 0.760 \\
\hline Admission mRS > $2(\%)$ & $27(15.4)$ & $6(4.3)$ & $21(58.3)$ & $<0.001^{*}$ \\
\hline \multicolumn{5}{|l|}{ Radiographic characteristics } \\
\hline \multicolumn{5}{|l|}{ Spetzler-Martin grade (\%) } \\
\hline I-II & $91(52.0)$ & $79(56.8)$ & $12(33.3)$ & $0.012^{*}$ \\
\hline III & $56(32.0)$ & $41(29.5)$ & $15(41.7)$ & 0.163 \\
\hline IV-V & $28(16.0)$ & $19(13.7)$ & $9(25.0)$ & 0.098 \\
\hline Volume $\left(\mathrm{cm}^{3}\right)$, median (IQR) & $8.6(4.1-25.6)$ & $8.5(4.1-25.6)$ & $8.7(4.3-26.5)$ & 0.388 \\
\hline Deep location (\%) & $51(29.1)$ & $35(25.2)$ & $16(44.4)$ & $0.023^{*}$ \\
\hline Eloquent location (\%) & $91(52.0)$ & $68(48.9)$ & $23(63.9)$ & 0.109 \\
\hline Deep venous drainage (\%) & $60(34.3)$ & $42(30.2)$ & $18(50.0)$ & $0.026^{*}$ \\
\hline Associated aneurysms (\%) & $22(12.6)$ & $14(10.1)$ & $8(22.2)$ & 0.093 \\
\hline \multicolumn{5}{|l|}{ Clinical features, mean (SD) } \\
\hline Heart rate, bpm & $80(10)$ & $80.3(9.8)$ & $77.0(9.3)$ & 0.312 \\
\hline SBP (mm Hg) & $120(19)$ & $119.5(17.1)$ & $121.4(25.7)$ & 0.666 \\
\hline DBP (mm Hg) & $77(11)$ & $76.8(10.4)$ & $80.1(12.8)$ & 0.162 \\
\hline BMI $\left(\mathrm{kg} / \mathrm{m}^{2}\right)$ & $22.5(4.5)$ & $22.6(4.5)$ & $22.2(4.5)$ & 0.654 \\
\hline \multicolumn{5}{|l|}{ Laboratory results, median (IQR) } \\
\hline Glucose $(\mathrm{mmol} / \mathrm{L})$ & $4.7(4.4-5.1)$ & $4.7(4.4-5.1)$ & $4.9(4.3-5.2)$ & $0.034^{*}$ \\
\hline Albumin $(\mathrm{g} / \mathrm{L})$ & $43.9(41.4-46.1)$ & $44.3(41.4-46.1)$ & $42.5(41.1-46.2)$ & $0.019^{*}$ \\
\hline Creatinine $(\mu \mathrm{mol} / \mathrm{L})$ & $58.5(46.5-68.3)$ & $58.6(46.5-68.3)$ & $55.7(46.8-68.2)$ & 0.591 \\
\hline $\mathrm{UA}(\mu \mathrm{mol} / \mathrm{L})$ & $309.7(254.9-363.6)$ & $314.0(254.9-362.2)$ & $302.2(247.9-362.9)$ & 0.269 \\
\hline $\mathrm{TC}(\mathrm{mmol} / \mathrm{L})$ & $4.2(3.6-4.8)$ & $4.3(3.6-4.8)$ & $4.1(3.6-4.9)$ & 0.072 \\
\hline $\mathrm{TG}(\mathrm{mmol} / \mathrm{L})$ & $1.0(0.7-1.5)$ & $1.0(0.7-1.5)$ & $1.0(0.7-1.5)$ & 0.622 \\
\hline HDL-C (mmol/L) & $1.2(1.0-1.5)$ & $1.2(1.0-1.5)$ & $1.2(1.0-1.5)$ & 0.480 \\
\hline LDL-C (mmol/L) & $2.6(2.0-3.1)$ & $2.6(2.0-3.1)$ & $2.4(1.9-3.1)$ & 0.191 \\
\hline ApoA (g/L) & $1.3(1.1-1.4)$ & $1.3(1.1-1.4)$ & $1.2(1.1-1.4)$ & 0.690 \\
\hline ApoB (g/L) & $0.8(0.7-1.0)$ & $0.8(0.7-1.0)$ & $0.8(0.7-1.0)$ & 0.203 \\
\hline Hcy $(\mu \mathrm{mol} / \mathrm{L})$ & $12.5(9.9-16.6)$ & $13.0(9.9-16.6)$ & $10.2(9.8-15.9)$ & $0.023^{*}$ \\
\hline HHcy (\%) & $57(32.6)$ & $49(35.3)$ & $8(22.2)$ & 0.137 \\
\hline Treatment modality & & & & 0.137 \\
\hline Microsurgery involvement & $78(44.6)$ & $58(41.7)$ & $20(55.6)$ & \\
\hline Nonmicrosurgery involvement & $97(55.4)$ & $81(58.3)$ & $16(44.4)$ & \\
\hline
\end{tabular}

bAVM: brain arteriovenous malformation; SD: standard deviation; mRS: modified Rankin Scale; IQR: interquartile range; SBP: systolic blood pressure; DBP: diastolic blood pressure; BMI: body mass index; UA: uric acid; TC: total cholesterol; TG: triglyceride; HDL-C: high-density lipoprotein cholesterol; LDL-C: lowdensity lipoprotein cholesterol; ApoA: apolipoprotein A; ApoB: apolipoprotein B; Hcy: homocysteine; HHcy: hyperhomocysteinemia. ${ }^{\#}$ Group 1, modified Rankin Scale $[\mathrm{mRS}] \leq 2$. Group $2, \mathrm{mRS}>2$. $^{*} P<0.05$, significant difference. 
poor neurological status $(\mathrm{mRS}>2)(P<0.001)$. In terms of the radiographic characteristics, the proportion of low SM grade (I-II) was found a significant difference between groups $(P=0.012)$. Patients with unfavorable outcomes were more located in deep areas $(P=0.023)$ and drained by deep veins $(P=0.026)$. In addition, higher level of glucose $(P=0.034)$, lower level of albumin $(P=0.019)$, and Hcy $(P=0.023)$ were found in Group 2.

3.2. Characteristics of bAVM Patients according to Hcy Quartiles. Clinical variables according to Hcy quartiles are summarized in Table 2. A linear association was observed between Hcy level and male sex $(P<0.001)$. Serum Hcy level was also related to age $(P=0.011)$, cigarette smoking $(P=0.001)$, and seizure manifestation $(P=0.034)$. Furthermore, Hcy was correlated with short-term neurological outcome $(P=0.027)$. Although the hemorrhagic risk was not a significant difference between Hcy quartiles $(P=0.052)$, a relatively lower incidence of rupture occurred in the groups of higher Hcy level (Q1: 61.4\%; Q2, 59.1\%; Q3, 58.1\%; and Q4, 47.7\%). Other variables were similar across groups $(P>0.05$ for all).

3.3. Analysis of Neurological Outcomes. Neurological outcomes of bAVM patients were analyzed. Thirty-six patients $(20.6 \%)$ were disabled (mRS $>2)$ at discharge, with no significant difference compared with admission $(P=0.211)$ (Figure 2). No in-hospital mortality occurred during hospitalization. According to the variation of $\mathrm{mRS}$ scores, most patients experienced an improved or unchanged neurological status in the short-term $(81.7 \%, n=143)$.

Risk factors for the neurological outcome of bAVM patients were identified (Table 3). Univariate analysis showed that hemorrhagic presentation, admission mRS score, AVM volume, deep location, deep venous drainage, associated aneurysms, glucose, albumin, TC, and Hcy were related to the neurological outcome. After additionally adjusting for age and male sex, hemorrhagic presentation (OR [odds ratios] 5.360, 95\% CI [confidence intervals] 1.208-23.790; $P=0.027$ ), admission $\mathrm{mRS}$ score (OR 2.225, 95\% CI 1.402-3.530; $P=0.001$ ), AVM volume (OR 1.024, 95\% CI 1.004-1.046; $P=0.021$ ), deep venous drainage (OR 8.813, 95\% CI 1.965-39.534; $P=0.004$ ), associated aneurysms (OR 5.711, 95\% CI 1.389-23.488; $P=0.016$ ), glucose (OR 1.897, 95\% CI 1.115-3.229; $P=0.018$ ), and Hcy (OR 0.838, 95\% CI $0.720-0.976 ; P=0.023$ ) were shown to be significantly associated with short-term disability in the multivariate analysis.

\section{Discussion}

Our retrospective study demonstrated the potential for serum levels of Hcy to serve as an objective biomarker for prognosticating short-term neurological outcomes in patients with bAVMs. The lower serum Hcy level predicted a higher risk of an unfavorable outcome. Furthermore, we identified a positive correlation between HHcy and symptomatic seizures on admission.

Admittedly, previously verified factors, including hemorrhagic presentation, admission mRS score, AVM volume, deep venous drainage, and associated aneurysms, have been broadly studied $[1,16]$. Sometimes, the evaluation between such indicators and prognosis will be subjective. When the classic Spetzler-Martin (SM) grading system [17], supplementary scale [18], and even with evolving fMRI-based HDVL grading scale combined with lesion-to eloquence distance (LED) are added [19], the evaluation becomes accurate but tedious at the same time. And there are some objectively advanced biomarkers, such as S100B, matrix metalloproteinase-9 (MMP-9), interleukin-1 beta (IL-1 $\beta$ ), vascular endothelial growth factor (VEGF), and N6-methyladenosine methyltransferase 3 (METTL3) [12, 20-22], which were proposed to be the predictors for the in-hospital complications and neurological outcomes. However, these indicators were unable to directly provide guidance for clinical practice. The underlying diagnostic and predictable values of laboratory biomarkers routinely examined after admission are required to be further excavated. To our best knowledge, we first associated the serum Hcy level with the short-term neurological outcome. After being adjusted for other known factors, the predicted value would still be significant (OR 0.838, 95\% CI 0.720 $0.976 ; P=0.023)$.

For decades, the abnormal Hcy level was acknowledged as the independent risk factors for the onset of ischemic stroke in several large scale multicenter trials [3, 5, 23, 24]. Afterward, the relationship between hemorrhagic stroke and HHcy has been subsequently studied but reached contrary conclusions. Li et.al found that elevated Hcy was correlated with hemorrhagic stroke by1.94-fold compared to the controls [5]. Larger hematoma volume in patients with thalamoganglionic intracerebral hemorrhage (ICH) associated with the elevated $\mathrm{Hcy}$ level was identified by $\mathrm{Hu}$ et al. [25]. Furthermore, Zhang and his colleagues developed a model consisted of Hcy level to identify high-risk groups for predicting recurrent $\mathrm{ICH}$, which can facilitate the preemptive clinical intervention [26]. Zhou et al. conducted a meta-analysis including $667 \mathrm{ICH}$ patients and demonstrated that the Hcy level is positively associated with a high risk of ICH [6]. Nevertheless, two cohorts in China found that $\mathrm{HHcy}$ correlated with a lower risk of hemorrhagic stroke [27]. For other hemorrhagic subtypes, such as subarachnoid hemorrhage $(\mathrm{SAH})$, researchers gained different findings. In 2001, McEvoy et al. revealed no association between the Hcy level and the etiology of SAH [28]. In favor of the upper conclusion, Grobelny et al. found no correlations between Hcy and delayed cerebral ischemia (DCI), while the gainof-function polymorphisms of the cystathionine $\beta$-synthase (CBS) gene could reduce the risk of DCI after aneurysmal SAH and improve the outcome at discharge [29]. Moreover, the CARAS study showed that increased CBS activity may exert its neuroprotective effects in altering the Hcy level and then improve clinical outcomes [30]. Another gene polymorphism methylenetetrahydrofolate reductase (MTHFR) C677T might contribute to a higher Hcy level to impair the clinical outcomes in patients with SAH [31]. Unlike their predecessors, Dhandapani et al. explored a reverse epidemiology paradox, where the higher Hcy level appears to be a significant association with both survival and favorable neurological outcomes [32]. 
TABLE 2: Characteristics of bAVM patients according to Hcy quartiles.

\begin{tabular}{|c|c|c|c|c|c|c|}
\hline \multirow{2}{*}{ Variables } & \multicolumn{5}{|c|}{ Hcy quartiles $^{\mathrm{a}}(\mu \mathrm{mol} / \mathrm{L})$} & \multirow{2}{*}{$P$ trend } \\
\hline & All $(n=175)$ & Q1 $(n=44)$ & $\mathrm{Q} 2(n=44)$ & Q3 $(n=43)$ & $\mathrm{Q} 4(n=44)$ & \\
\hline Age (y), mean (SD) & $29.6(14.7)$ & $24.0(15.7)$ & $30.8(14.2)$ & $34.4(13.4)$ & $29.3(13.8)$ & $0.011^{*}$ \\
\hline Sex, male (\%) & $100(57.1)$ & $13(29.5)$ & $22(50.0)$ & $29(67.4)$ & $36(81.8)$ & $<0.001^{*}$ \\
\hline \multicolumn{7}{|l|}{ Medical history (\%) } \\
\hline Hypertension & $14(8.0)$ & $2(4.5)$ & $2(4.5)$ & $6(14.0)$ & $4(9.1)$ & 0.210 \\
\hline Diabetes mellitus & $5(2.9)$ & $1(2.3)$ & $1(2.3)$ & $1(2.3)$ & $2(4.5)$ & 0.540 \\
\hline Hyperlipidemia & $1(0.6)$ & $0(0)$ & $0(0)$ & $1(2.3)$ & $0(0)$ & 0.653 \\
\hline Cigarette smoking & $31(17.7)$ & $1(2.3)$ & $7(15.9)$ & $10(23.3)$ & $13(29.5)$ & $0.001^{*}$ \\
\hline Alcohol drinking & $17(9.7)$ & $0(0)$ & $4(9.1)$ & $9(20.9)$ & $4(9.1)$ & 0.052 \\
\hline \multicolumn{7}{|l|}{ Primary symptom (\%) } \\
\hline Hemorrhage & $99(56.6)$ & $27(61.4)$ & $26(59.1)$ & $25(58.1)$ & $21(47.7)$ & 0.210 \\
\hline Seizure & $35(20.0)$ & $5(11.4)$ & $8(18.2)$ & $9(20.9)$ & $13(29.5)$ & $0.034^{*}$ \\
\hline Neurological dysfunction & $31(17.7)$ & $9(20.5)$ & $8(18.2)$ & $8(18.6)$ & $6(13.6)$ & 0.416 \\
\hline Admission mRS > $2(\%)$ & $27(15.4)$ & $13(29.5)$ & $3(6.8)$ & $6(14.0)$ & $5(11.4)$ & 0.052 \\
\hline \multicolumn{7}{|l|}{ AVM characteristics } \\
\hline Spetzler-Martin grade (\%) & & & & & & 0.885 \\
\hline I-II & $91(52.0)$ & $19(43.2)$ & $27(61.4)$ & $23(53.5)$ & $22(50.0)$ & \\
\hline III & $56(32.0)$ & $17(38.6)$ & $12(27.3)$ & $13(30.2)$ & $14(31.8)$ & \\
\hline IV-V & $28(16.0)$ & $8(18.2)$ & $5(11.4)$ & $7(16.3)$ & $8(18.2)$ & \\
\hline Volume $\left(\mathrm{cm}^{3}\right)$, median (IQR) & $8.6(4.1-25.6)$ & $11.4(5.0-29.7)$ & $7.3(4.5-18.7)$ & $8.6(3.1-22.7)$ & $11.5(4.1-31.8)$ & 0.596 \\
\hline Deep location (\%) & $51(29.1)$ & $13(29.5)$ & $11(25.0)$ & $12(27.9)$ & $15(34.1)$ & 0.338 \\
\hline Eloquent location (\%) & $91(52.0)$ & $26(59.1)$ & $21(47.7)$ & $23(53.5)$ & $21(47.7)$ & 0.400 \\
\hline Deep venous drainage (\%) & $60(34.3)$ & $15(34.1)$ & $13(29.5)$ & $16(37.2)$ & $16(36.4)$ & 0.687 \\
\hline Associated aneurysms (\%) & $22(12.6)$ & $6(13.6)$ & $5(11.4)$ & $6(14.0)$ & $5(11.4)$ & 0.849 \\
\hline Microsurgery involvement (\%) & $78(44.5)$ & $23(52.3)$ & $19(43.2)$ & $18(41.9)$ & $18(40.9)$ & 0.145 \\
\hline Postoperative stroke (\%) & $13(7.4)$ & $5(11.4)$ & $1(2.3)$ & $6(14.0)$ & $1(2.3)$ & 0.373 \\
\hline Short-term neurological disability (\%) & $36(20.6)$ & $17(38.6)$ & $4(9.1)$ & $9(20.9)$ & $6(13.6)$ & $0.027^{*}$ \\
\hline
\end{tabular}

bAVM: brain arteriovenous malformation; Hcy: homocysteine; SD: standard deviation; mRS: modified Rankin Scale; IQR: interquartile range. ${ }^{*} P<0.05$, significant difference. ${ }^{a}$ Serum levels of Hcy in quartiles: Q1, $<9.9 \mu \mathrm{mol} / \mathrm{L}$; Q2, 9.9-12.7 $\mu \mathrm{mol} / \mathrm{L} ; \mathrm{Q} 3,12.8-16.6 \mu \mathrm{mol} / \mathrm{L}$; and Q4, $\geq 16.1 \mu \mathrm{mol} / \mathrm{L}$.

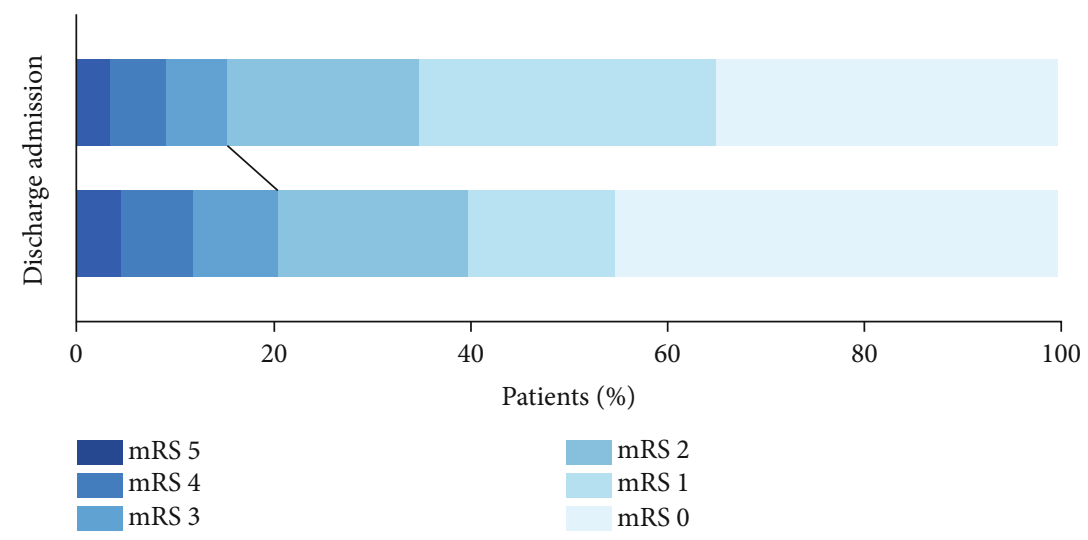

FIGURE 2: Comparison of mRS score in bAVM patients at admission and discharge. Thirty-six patients $(20.6 \%)$ were disabled (mRS $>2)$ at discharge, with no significant difference compared with admission $(P=0.211)$. mRS: modified Rankin Scale.

In the present study, we reached a similar conclusion as SAH groups. Higher serum Hcy levels seemed to be a survival advantage in patients with bAVMs. We supposed that the lower serum Hcy level attributed to the activation in the cas- cade usage of Hcy's endogenously thrombotic effect after the hemorrhagic bAVM. As an intermediate metabolic product in the circle involving folate and vitamins, Hcy could be modifiable [33]. Two randomized controlled trials showed 
TABLE 3: Logistic regression analysis on the neurological outcome.

\begin{tabular}{|c|c|c|c|c|c|c|}
\hline \multirow{2}{*}{ Variables } & \multicolumn{3}{|c|}{ Univariate analysis } & \multicolumn{3}{|c|}{ Multivariate analysis } \\
\hline & OR & $95 \% \mathrm{CI}$ & $P$ value & OR & $95 \% \mathrm{CI}$ & $P$ value \\
\hline Age & 0.994 & $0.969-1.019$ & 0.633 & 1.011 & $0.969-1.055$ & 0.609 \\
\hline Male sex & 1.229 & $0.581-2.599$ & 0.590 & 2.528 & $0.659-9.698$ & 0.177 \\
\hline \multicolumn{7}{|l|}{ Medical history } \\
\hline Hypertension & 2.330 & $0.729-7.441$ & 0.153 & & & \\
\hline Diabetes mellitus & 2.667 & $0.429-16.594$ & 0.293 & & & \\
\hline Cigarette smoking & 0.912 & $0.343-2.424$ & 0.853 & & & \\
\hline Alcohol drinking & 1.212 & $0.370-3.966$ & 0.751 & & & \\
\hline \multicolumn{7}{|l|}{ Primary symptoms } \\
\hline Hemorrhage & 4.084 & $1.677-9.943$ & 0.002 & 5.360 & $1.208-23.790$ & $0.027^{*}$ \\
\hline Seizure & 0.586 & $0.210-1.637$ & 0.308 & & & \\
\hline Neurological dysfunction & 1.157 & $0.454-2.947$ & 0.760 & & & \\
\hline Admission mRS score & 2.496 & $1.803-3.457$ & $<0.001$ & 2.225 & $1.402-3.530$ & $0.001^{*}$ \\
\hline \multicolumn{7}{|l|}{ AVM characteristics } \\
\hline Volume & 1.018 & $1.005-1.032$ & 0.007 & 1.024 & $1.004-1.046$ & $0.021^{*}$ \\
\hline Deep location & 2.377 & $1.111-5.087$ & 0.026 & 0.927 & $0.238-3.613$ & 0.913 \\
\hline Eloquent location & 1.847 & $0.866-3.938$ & 0.112 & & & \\
\hline Deep venous drainage & 2.310 & $1.094-4.874$ & 0.028 & 8.813 & $1.965-39.534$ & $0.004^{*}$ \\
\hline Associated aneurysms & 2.551 & $0.976-6.666$ & 0.056 & 5.711 & $1.389-23.488$ & $0.016^{*}$ \\
\hline \multicolumn{7}{|l|}{ Clinical features } \\
\hline Heart rate & 0.980 & $0.944-1.019$ & 0.311 & & & \\
\hline SBP & 1.004 & $0.985-1.025$ & 0.664 & & & \\
\hline DBP & 1.024 & 0.990-1.059 & 0.164 & & & \\
\hline BMI & 0.980 & $0.899-1.069$ & 0.652 & & & \\
\hline \multicolumn{7}{|l|}{ Laboratory results } \\
\hline Glucose & 1.579 & $1.151-2.166$ & 0.005 & 1.897 & $1.115-3.229$ & $0.018^{*}$ \\
\hline Albumin & 0.900 & $0.816-0.993$ & 0.035 & 0.968 & $0.820-1.142$ & 0.697 \\
\hline Creatinine & 0.995 & $0.972-1.018$ & 0.667 & & & \\
\hline UA & 1.001 & 0.999-1.002 & 0.325 & & & \\
\hline $\mathrm{TC}$ & 0.643 & $0.421-0.983$ & 0.042 & 0.194 & $0.033-1.129$ & 0.068 \\
\hline TG & 0.944 & $0.636-1.403$ & 0.776 & & & \\
\hline HDL-C & 1.639 & $0.870-3.088$ & 0.126 & & & \\
\hline LDL-C & 0.692 & $0.441-1.086$ & 0.109 & & & \\
\hline ApoA & 0.868 & $0.228-3.303$ & 0.836 & & & \\
\hline ApoB & 0.253 & $0.040-1.595$ & 0.143 & & & \\
\hline Hcy & 0.937 & $0.878-1.001$ & 0.055 & 0.838 & $0.720-0.976$ & $0.023^{*}$ \\
\hline Microsurgery involvement & 1.746 & $0.834-3.654$ & 0.139 & & & \\
\hline
\end{tabular}

OR: odds ratio; CI: confidence intervals; AVM: arteriovenous malformation; SBP: systolic blood pressure; DBP: diastolic blood pressure; BMI: body mass index; UA: uric acid; TC: total cholesterol; TG: triglyceride; HDL-C: high-density lipoprotein cholesterol; LDL-C: low-density lipoprotein cholesterol; ApoA: apolipoprotein A; ApoB: apolipoprotein B; Hcy: homocysteine. ${ }^{*} P<0.05$, significant difference.

that folic acid/vitamin B12 or vitamin B6 had no effect of treatment on total mortality [3,4]. However, Wang et al. performed a meta-analysis showing effective reduction in patients with stroke in primary prevention [34]. The conflicts between these studies were an account of the baseline folate consumption. The management of oral nutritional supplements on Hcy in the bAVMs group needs further investigation.

Another independent prominent predictor for the unfavorable neurological outcome is the higher serum glucose level (OR 1.897, 95\% CI 1.115-3.229; $P=0.018$ ). The same results were reached in the hemorrhagic subtypes, including ICH and SAH $[35,36]$.

Some clinical features of bAVMs determined by this study differed from those in previous reports. We demonstrated a statistical significance between Hcy quartiles and seizure manifestation $(P=0.034)$, which can induce unfavorable outcomes at discharge in the patients with bAVMs. The relationship between Hcy and seizure was first proposed in 
an intrathecal chemotherapy-treated boy who suffered from leukemia [37]. And any transient elevation of Hcy may be related to the seizure risk $[38,39]$. Although it is hard to determine whether the elevated Hcy is the cause or the result of a seizure, we offered neurosurgeons a new insight into the prophylactic and off-label utility of antiepileptic drugs.

There are some limitations that should be acknowledged. First, due to the nature of the respective study, we are aware of the fact that its design is one of the considerations which should be improved in the future. This study's small sample size is the second considerable limitation because larger samples are preferred for relevant studies. As such, findings should be interpreted with caution until further high-level prospective studies or larger data sets are available. Third, the baseline dietary and folate status of the patients were undocumented. Though the population was under established policies of population folate supplementation for the few decades nationwide, we enrolled patients before the policy was carried out, which could be a confounding variable. Fourth, the outcomes were only measured at the discharge point and were not conducted at the 3-month follow-up. This is our ongoing study of enrolled patients, and we expect to make some progress in the recent future. Further, longer follow-up studies should be conducted to demonstrate the clinical relevance between the serum Hcy level and longterm outcomes.

\section{Conclusions}

In conclusion, our results indicated that the lower serum Hcy level is strongly associated with in-hospital unfavorable outcomes. Further prospective studies of Hcy natural history and management in the bAVMs groups are needed, which would be valuable for evaluating the disease-modifying efficacy of oral nutritional supplements in bAVM patients.

\section{Data Availability}

The data used to support the findings of this study are available from the corresponding author upon reasonable request.

\section{Conflicts of Interest}

The authors declare that they have no conflicts of interest.

\section{Authors' Contributions}

Fa Lin, Chaofan Zeng, and Peicong Ge contributed equally to this work.

\section{Acknowledgments}

This study was supported by the National Key Technologies Research and Development Program of China (2016YFC 1301800), Beijing Science and Technology Supporting Plan (D161100003816005), and Beijing Municipal Administration of Hospitals' Mission Plan (SML20150501).

\section{References}

[1] R. M. Friedlander, "Arteriovenous malformations of the brain," The New England Journal of Medicine, vol. 356, no. 26, pp. 2704-2712, 2007.

[2] S. I. Nikolaev, S. Vetiska, X. Bonilla et al., "Somatic ActivatingKRASMutations in arteriovenous malformations of the brain," The New England Journal of Medicine, vol. 378, no. 3, pp. 250-261, 2018.

[3] M. V. Holmes, P. Newcombe, J. A. Hubacek et al., "Effect modification by population dietary folate on the association between_MTHFR_genotype, homocysteine, and stroke risk: a meta-analysis of genetic studies and randomised trials," The Lancet, vol. 378, no. 9791, pp. 584-594, 2011.

[4] M. Ebbing, Ø. Bleie, P. M. Ueland et al., "Mortality and cardiovascular events in patients treated with homocysteinelowering $B$ vitamins after coronary angiography: a randomized controlled trial," JAMA, vol. 300, no. 7, pp. 795-804, 2008.

[5] Z. Li, L. Sun, H. Zhang et al., "Elevated plasma homocysteine was associated with hemorrhagic and ischemic stroke, but methylenetetrahydrofolate reductase gene C677T polymorphism was a risk factor for thrombotic stroke: a multicenter case-control study in China," Stroke, vol. 34, no. 9, pp. 20852090, 2003.

[6] Z. Zhou, Y. Liang, H. Qu et al., "Plasma homocysteine concentrations and risk of intracerebral hemorrhage: a systematic review and meta-analysis," Scientific Reports, vol. 8, no. 1, p. 2568, 2018.

[7] V. V. Aleksandrin, A. V. Ivanov, E. D. Virus, P. O. Bulgakova, and A. A. Kubatiev, "Application of wavelet analysis to detect dysfunction in cerebral blood flow autoregulation during experimental hyperhomocysteinaemia," Lasers in Medical Science, vol. 33, no. 6, pp. 1327-1333, 2018.

[8] M. Hatefi, S. Behzadi, M. M. Dastjerdi et al., "Correlation of homocysteine with cerebral hemodynamic abnormality, endothelial dysfunction markers, and cognition impairment in patients with traumatic brain injury," World Neurosurgery, vol. 97, pp. 70-79, 2017.

[9] M.-H. Lim, Y. I. Cho, and S.-K. Jeong, "Homocysteine and pulsatility index of cerebral arteries," Stroke, vol. 40, no. 10, pp. 3216-3220, 2009.

[10] T. Hashimoto, G. Wen, M. T. Lawton et al., "Abnormal expression of matrix metalloproteinases and tissue inhibitors of metalloproteinases in brain arteriovenous malformations," Stroke, vol. 34, no. 4, pp. 925-931, 2003.

[11] H. Min, J. Hong, I.-H. Cho et al., "TLR2-induced astrocyte MMP9 activation compromises the blood brain barrier and exacerbates intracerebral hemorrhage in animal models," Molecular Brain, vol. 8, no. 1, p. 23, 2015.

[12] E. Shotar, C. Amouyal, A. Jacquens et al., "S100B serum elevation predicts in-hospital mortality after brain arteriovenous malformation rupture," Stroke, vol. 50, no. 5, pp. 1250-1253, 2019.

[13] N. Tyagi, W. Gillespie, J. C. Vacek, U. Sen, S. C. Tyagi, and D. Lominadze, "Activation of GABA-A receptor ameliorates homocysteine-induced MMP-9 activation by ERK pathway," Journal of Cellular Physiology, vol. 220, no. 1, pp. 257-266, 2009.

[14] I. Wiernicki, B. Millo, K. Safranow, B. Gorecka-Szyld, and P. Gutowski, "MMP-9, homocysteine and CRP circulating levels are associated with intraluminal thrombus thickness of 
abdominal aortic aneurysms-new implication of the old biomarkers," Disease Markers, vol. 31, no. 2, pp. 67-74, 2011.

[15] Q. Wang, J. Zhang, K. Zhao, and B. Xu, "Hyperhomocysteinemia is an independent risk factor for intracranial aneurysms: a case-control study in a Chinese Han population," Neurosurgical Review, vol. 43, no. 4, pp. 1127-1134, 2020.

[16] C. S. Ogilvy, P. E. Stieg, I. Awad et al., "Recommendations for the management of intracranial arteriovenous malformations: a statement for healthcare professionals from a special writing group of the Stroke Council, American Stroke Association," Circulation, vol. 103, no. 21, pp. 2644-2657, 2001.

[17] R. F. Spetzler and N. A. Martin, "A proposed grading system for arteriovenous malformations," Journal of Neurosurgery, vol. 65, no. 4, pp. 476-483, 1986.

[18] M. T. Lawton, H. Kim, C. E. McCulloch, B. Mikhak, and W. L. Young, "A supplementary grading scale for selecting patients with brain arteriovenous malformations for surgery," Neurosurgery, vol. 66, no. 4, pp. 702-713, 2010.

[19] Y. Jiao, F. Lin, J. Wu et al., "A supplementary grading scale combining lesion-to-eloquence distance for predicting surgical outcomes of patients with brain arteriovenous malformations," Journal of Neurosurgery, vol. 128, no. 2, pp. 530-540, 2018.

[20] H. Kim, L. Pawlikowska, Y. Chen, H. Su, G. Y. Yang, and W. L. Young, "Brain arteriovenous malformation biology relevant to hemorrhage and implication for therapeutic development," Stroke, vol. 40, no. 3, Supplement 1, pp. S95-S97, 2009.

[21] L. J. Wang, Y. Xue, R. Huo et al., "N6-methyladenosine methyltransferase METTL3 affects the phenotype of cerebral arteriovenous malformation via modulating Notch signaling pathway," Journal of Biomedical Science, vol. 27, no. 1, p. 62, 2020.

[22] M. Xu, X. Liu, G. Mei, J. Zhang, W. Wang, and H. Xu, "Radiosurgery reduces plasma levels of angiogenic factors in brain arteriovenous malformation patients," Brain Research Bulletin, vol. 140, pp. 220-225, 2018.

[23] G. Boysen, T. Brander, H. Christensen, R. Gideon, and T. Truelsen, "Homocysteine and risk of recurrent stroke," Stroke, vol. 34, no. 5, pp. 1258-1261, 2003.

[24] H. Iso, Y. Moriyama, S. Sato et al., "Serum total homocysteine concentrations and risk of stroke and its subtypes in Japanese," Circulation, vol. 109, no. 22, pp. 2766-2772, 2004.

[25] F. Zhou, B. Chen, C. Chen et al., "Elevated homocysteine levels contribute to larger hematoma volume in patients with intracerebral hemorrhage," Journal of Stroke and Cerebrovascular Diseases, vol. 24, no. 4, pp. 784-788, 2015.

[26] S. Zhang, X. Zhang, Y. Ling, and A. Li, "Predicting recurrent hypertensive intracerebral hemorrhage: derivation and validation of a risk-scoring model based on clinical characteristics," World Neurosurgery, vol. 127, pp. e162-e171, 2019.

[27] T. H. T. Chiu, H.-R. Chang, L.-Y. Wang, C.-C. Chang, M.N. Lin, and C.-L. Lin, "Vegetarian diet and incidence of total, ischemic, and hemorrhagic stroke in 2 cohorts in Taiwan," Neurology, vol. 94, no. 11, pp. e1112-e1121, 2020.

[28] A. W. McEvoy, C. Marras, N. D. Kitchen, and A. Briddon, "Plasma total homocysteine and subarachnoid haemorrhage in a co-factor replete population," Amino Acids, vol. 21, no. 3, pp. 237-241, 2001.

[29] B. T. Grobelny, A. F. Ducruet, P. A. DeRosa et al., "Gain-offunction polymorphisms of cystathionine $\beta$-synthase and delayed cerebral ischemia following aneurysmal subarachnoid hemorrhage," Journal of Neurosurgery, vol. 115, no. 1, pp. 101107, 2011.

[30] P. Hendrix, P. M. Foreman, M. R. Harrigan et al., “Association of cystathionine beta-synthase polymorphisms and aneurysmal subarachnoid hemorrhage," Journal of Neurosurgery, vol. 128, no. 6, pp. 1771-1777, 2018.

[31] M. Kumar, S. Goudihalli, K. Mukherjee, S. Dhandapani, and R. Sandhir, "Methylenetetrahydrofolate reductase C677T variant and hyperhomocysteinemia in subarachnoid hemorrhage patients from India," Metabolic Brain Disease, vol. 33, no. 5, pp. 1617-1624, 2018.

[32] S. Dhandapani, S. Goudihalli, K. K. Mukherjee et al., "Prospective study of the correlation between admission plasma homocysteine levels and neurological outcome following subarachnoid hemorrhage: a case for the reverse epidemiology paradox?," Acta Neurochirurgica, vol. 157, no. 3, pp. 399-407, 2015.

[33] P. Ge, Q. Zhang, X. Ye et al., "Modifiable risk factors associated with Moyamoya disease," Stroke, vol. 51, no. 8, pp. 2472-2479, 2020.

[34] X. Wang, X. Qin, H. Demirtas et al., "Efficacy of folic acid supplementation in stroke prevention: a meta-analysis," The Lancet, vol. 369, no. 9576, pp. 1876-1882, 2007.

[35] S. Hafez, M. Abdelsaid, S. El-Shafey, M. H. Johnson, S. C. Fagan, and A. Ergul, "Matrix metalloprotease 3 exacerbates hemorrhagic transformation and worsens functional outcomes in hyperglycemic stroke," Stroke, vol. 47, no. 3, pp. 843-851, 2016.

[36] F. Schlenk, A. Nagel, D. Graetz, and A. S. Sarrafzadeh, "Hyperglycemia and cerebral glucose in aneurysmal subarachnoid hemorrhage," Intensive Care Medicine, vol. 34, no. 7, pp. 1200-1207, 2008.

[37] C. T. Quinn, J. C. Griener, T. Bottiglieri, and B. A. Kamen, "Methotrexate, homocysteine, and seizures," Journal of Clinical Oncology, vol. 16, no. 1, pp. 393-394, 1998.

[38] R. He, R. Mo, M. Shen et al., "Variable phenotypes and outcomes associated with the MMACHC c.609G $>$ A homologous mutation: long term follow-up in a large cohort of cases," Orphanet Journal of Rare Diseases, vol. 15, no. 1, p. 200, 2020.

[39] S. Kishi, J. Griener, C. Cheng et al., "Homocysteine, pharmacogenetics, and neurotoxicity in children with leukemia," Journal of Clinical Oncology, vol. 21, no. 16, pp. 3084-3091, 2003. 